\title{
STUDY OF THE COINCIDENCES BETWEEN THE GRAVITATIONAL WAVE DETECTORS EXPLORER AND NAUTILUS IN THE YEAR 2001
}

P. Astone ${ }^{1}$,D. Babusci ${ }^{2}$, M. Bassan ${ }^{3}$, P. Bonifazi ${ }^{4}$, P. $_{\text {Carelli }}{ }^{5}$, G. Cavallari $^{6}$, E. Coccia ${ }^{3}$, C. Cosmelli ${ }^{7}$, S.D'Antonio ${ }^{3}$, V. Fafone ${ }^{2}$, G.Federici ${ }^{7}$, S.Frasca ${ }^{7}$, G. Giordano ${ }^{2}$,

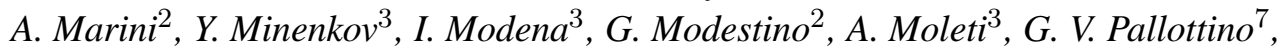
G. Pizzella ${ }^{8}$, L. Quintieri ${ }^{2}$, A.Rocchi ${ }^{3}$, F. Ronga ${ }^{2}$, R. Terenzi $^{4}$, G.Torrioli $^{9}$, M. $_{\text {. }}$ Visco $^{4}$

\author{
1) Istituto Nazionale di Fisica Nucleare INFN, Rome \\ 2) Istituto Nazionale di Fisica Nucleare INFN, Frascati \\ 3) University of Rome "Tor Vergata" and INFN, Rome \\ 4) IFSI-CNR and INFN, Rome \\ 5) University of L'Aquila and INFN, Rome \\ 6) CERN, Geneva, Switzerland \\ 7) University of Rome "La Sapienza" and INFN, Rome \\ 8) University of Rome "Tor Vergata" and INFN, Frascati \\ 9) IESS-CNR, Rome
}

\begin{abstract}
We report the result from a search for bursts of gravitational waves using data collected by the cryogenic resonant detectors EXPLORER and NAUTILUS during the year 2001, for a total measuring time of 90 days. With these data we repeated the coincidence search performed on the 1998 data (which showed a small coincidence excess) applying data analysis algorithms based on known physical characteristics of the detectors. With the 2001 data a new interesting coincidence excess is found when the detectors are favorably oriented with respect to the Galactic Disk.
\end{abstract}

PACS:04.80,04.30

\section{Introduction}

Cryogenic gravitational wave (GW) antennas entered into long term data taking operation in 1990 (EXPLORER [1]), in 1991 (ALLEGRO [2]), in 1993 (NIOBE [3]), in 1994 (NAUTILUS [他) and in 1997 (AURIGA [5]), with gradual performance improvements over the years.

Analysis of the data taken in coincidence among all cryogenic resonant detectors in operation during the years 1997 and 1998 was performed [6]. No coincidence excess was found above background using the event lists produced under the protocol of the International Gravitational Event Collaboration (IGEC), among the groups ALLEGRO, AURIGA, EXPLORER / NAUTILUS and NIOBE.

Later [7], a coincidence search between the data of EXPLORER and NAUTILUS was carried out by introducing in the data analysis considerations based on physical characteristics of the detectors: the event energy and the directionality. The result was a small coincidence excess when the detectors were favorably oriented with respect to the Galactic Centre.

Here we extend our analysis to new data obtained in the year 2001, when both EXPLORER and NAUTILUS were operating at their best sensitivity, using the same procedures applied for the previous analysis [7]. As previously done in ref. [7] we shall sometimes use the word probability, although we are well aware that its significance might be jeopardized by any possible data selection. With this proviso we shall use probability estimations in comparing different experimental conditions. 
Table 1: Main characteristics of the two detectors in the year 2001. The axes of the two detectors are aligned to within a few degrees of one other, the chance of coincidence detection thus being maximized. The pulse sensitivity for both detectors is of the order of $h \sim 410^{-19}$ for 1 ms bursts.

\begin{tabular}{|c|c|c|c|c|c|c|c|}
\hline detector & latitude & longitude & azimuth & $\begin{array}{c}\text { mass } \\
\mathrm{kg}\end{array}$ & $\begin{array}{c}\text { frequencies } \\
\mathrm{Hz}\end{array}$ & $\begin{array}{c}\text { temperature } \\
\mathrm{K}\end{array}$ & $\begin{array}{c}\text { bandwidth } \\
\mathrm{Hz}\end{array}$ \\
\hline EXPLORER & $46.45 \mathrm{~N}$ & $6.20 \mathrm{E}$ & $39^{\circ} \mathrm{E}$ & 2270 & 904.7 & 2.6 & $\sim 9$ \\
& & & & & 921.3 & & \\
NAUTILUS & $41.82 \mathrm{~N}$ & \multirow{2}{*}{$12.67 \mathrm{E}$} & $44^{\circ} \mathrm{E}$ & 2270 & 906.97 & 1.5 & $\sim 0.4$ \\
& & & & & 922.46 & & \\
\hline
\end{tabular}

\section{Experimental data}

The resonant mass GW detectors NAUTILUS, operating at the INFN Frascati Laboratory, and EXPLORER, operating at CERN, both consist of an Aluminium $2270 \mathrm{~kg}$ bar cooled to very low temperatures. A resonant transducer converts the mechanical oscillations into an electrical signal and is followed by a dcSQUID electronic amplifier. The bar and the resonant transducer form a coupled oscillator system with two resonant modes.

With respect to the year 1998 the following changes were made in the set up of the detectors: the NAUTILUS detector operated at a thermodynamic temperature of $1.5 \mathrm{~K}$ instead of $0.14 \mathrm{~K}$; the EXPLORER detector was equipped with a new transducer providing a larger bandwidth and consequently enhanced sensitivity. The characteristics of the two detectors are given in the Table 1 .

The data, sampled at intervals of $12.8 \mathrm{~ms}$ for NAUTILUS and of $6.4 \mathrm{~ms}$ for EXPLORER, are filtered with an adaptive filter matched to delta-like signals for the detection of short bursts [8]. This search for bursts is suitable for any transient GW which shows a nearly flat Fourier spectrum at the two resonant frequencies of each detector. The metric perturbation $h$ can either be a millisecond pulse, a signal made by a few millisecond cycles, or a signal sweeping in frequency through the detector resonances. This search is therefore sensitive to different kinds of GW sources, such as a stellar gravitational collapse, the last stable orbits of an inspiraling neutron star or black hole binary, its merging and its final ringdown.

Let $x(t)$ be the filtered output of the detector. This quantity is normalized, using the detector calibration, such that its square gives the energy innovation of the oscillation for each sample, expressed in kelvin units.

For well behaved noise due only to the thermal motion of the oscillators and to the electronic noise of the amplifier, the distribution of $x(t)$ is normal with zero mean. Its variance (average value of the square of $x(t)$ ) is called ef fective temperature and is indicated with $T_{e f f}$. The distribution of $x(t)$ is

$$
f(x)=\frac{1}{\sqrt{2 \pi T_{e f f}}} e^{-\frac{x^{2}}{2 T_{e f f}}}
$$

In order to extract from the filtered data sequence events to be analyzed we set a threshold in terms of a critical ratio defined by

$$
C R=\frac{|x|-\overline{|x|}}{\sigma(|x|)}
$$

where $\sigma(|x|)$ is the standard deviation of $|x|$ and $\overline{|x|}$ the moving average, computed over the preceeding ten minutes.

The threshold is set at $\mathrm{CR}=6$ in order to obtain, in the presence of thermal and electronic noise alone, a reasonable number of events per day (see ref.[7]). This threshold corresponds to energy $E_{t}=$ $19.5 T_{\text {eff }}$. When $|x|$ goes above the threshold, its time behaviour is considered until it falls back below the threshold for longer than three seconds. The maximum amplitude and its occurrence time define the event. 


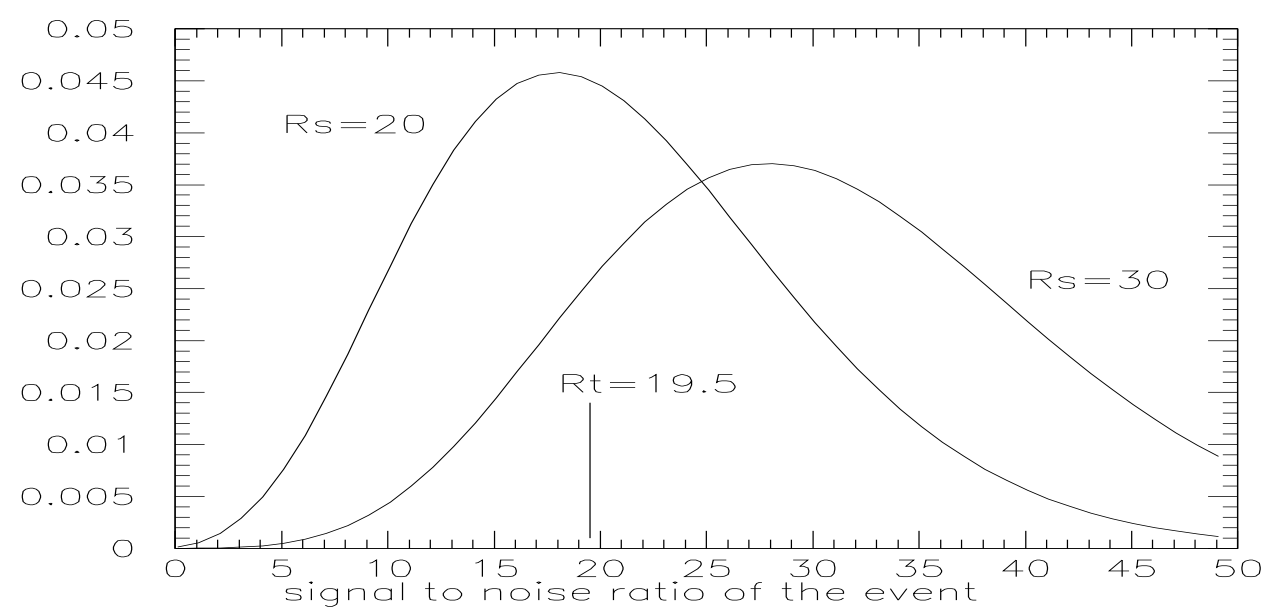

Fig. 1: Differential probability that the event has the signal-to-noise ratio shown on the abscissa when the signal has $R_{s}=20$ (near the threshold $R_{t}=19.5$ ) and $R_{s}=30$.

The searched events are the ones that are due to a combination of a GW signal of energy $E_{s}$ and of the noise. The theoretical probability to detect a signal with a given signal to noise ratio $R_{s}=\frac{E_{s}}{T_{e f f}}$, in the presence of a well behaved Gaussian noise is [9]

$$
\operatorname{probability}\left(R_{s}\right)=\int_{R_{t}}^{\infty} \frac{1}{\sqrt{2 \pi R_{e}}} e^{-\frac{\left(R_{s}+R_{e}\right)}{2}} \cosh \left(\sqrt{R_{e} \cdot R_{s}}\right) d R_{e}
$$

where $R_{e}$ is the signal to noise ratio for the event and $R_{t}=\frac{E_{t}}{T_{e f f}}=19.5$ for the EXPLORER and NAUTILUS detectors.

The behaviour of the integrand is shown in fig. 1. This figure shows the spread of the event energy due to noise for a given $R_{s}$ of the signal. It shows that signals with $R_{s}=20$ (near the threshold) have a probability of about 50\% not to be detected, and signals with $R_{s}=30$, rather larger than the threshold, still have a probability of near $15 \%$ not to be detected. The distinction between the two concepts, signal and event, is essential for our analysis, as discussed in ref [ד].

Computation of the GW amplitude $h$ from the energy signal $E_{s}$ requires a model for the signal shape. A conventionally chosen shape is a short pulse lasting a time of $\tau_{g}$, resulting (for optimal orientation, see later) in the relationship

$$
h=\frac{1}{4 L f^{2}} \frac{1}{\tau_{g}} \sqrt{\frac{k E_{s}}{M}}
$$

where $f$ is the resonance frequency, $\mathrm{L}$ and $\mathrm{M}$ the length and the mass of the bar and $\tau_{g}$ is conventionally assumed equal to $1 \mathrm{~ms}$ (for instance, for $E_{s}=1 \mathrm{mK}$ we have $h=2.510^{-19}$ ).

\section{Data selection}

All events which are in coincidence within a time window of $\pm 5 s$ with signals observed by a seismometer are eliminated. This criterion cuts about $8 \%$ of the events. 

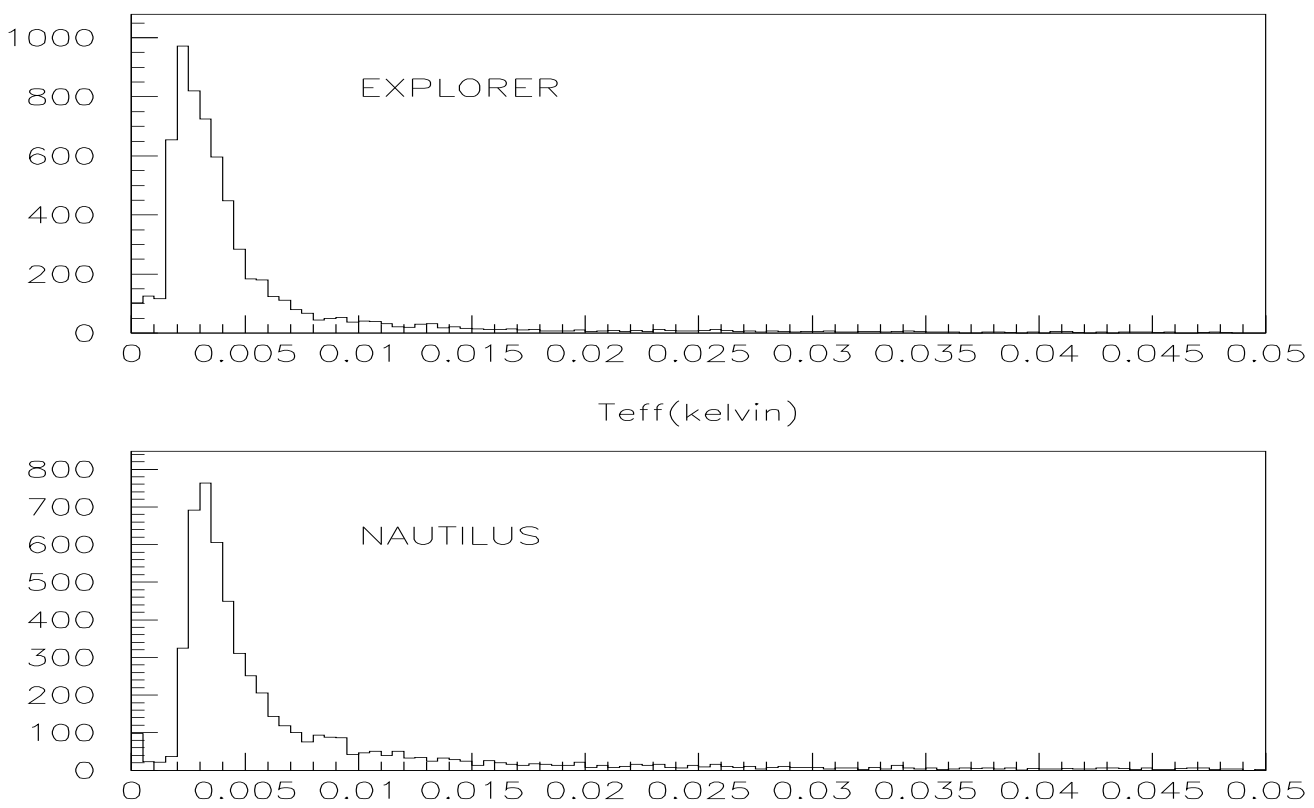

Fig. 2: The distributions of the hourly averages of $T_{e f f}$ in kelvin units for EXPLORER and NAUTILUS. We accept only the time periods with hourly averages $\overline{T_{e f f}} \leq 10 \mathrm{mK}$.

It is observed that the experimental data are affected by non gaussian noise which, in some cases, cannot be observed with any other auxiliary detector. Thus a strategy is needed to select periods during which the detectors operate in a satisfactory way, as discussed in the following paragraphs.

To this end we consider the quantity $T_{e f f}$, which we used in two ways. The first was to compute $T_{\text {eff }}$ by averaging $x^{2}$ over one hour of continuous measurements $\left(\overline{T_{e f f}}\right)$, the second to consider the $T_{e f f}$ averaged during the ten minutes preceeding each event. For the hourly averages we show the distribution in fig.2. On observing this figure we decided to consider for the search for coincidences only the time periods with hourly averages smaller than $10 \mathrm{mK}$. The distributions for the $T_{\text {eff }}$ averaged over the ten minutes preceeding each event are shown in fig. 3 for EXPLORER and NAUTILUS. It will be noticed that the number of events is larger for EXPLORER than for NAUTILUS. This depends on the bandwidth $\Delta f$ which is larger for EXPLORER(see Table 1).

On observing these distributions we decided, as conservative a priori data selection, to make a cut and accept only the events for which the corresponding $T_{\text {eff }}$ was below $7 \mathrm{mK}$ (and there was no seismometer veto). This meant regarding the bump at $10 \mathrm{mK}$, for NAUTILUS, as due to extra noise. We recall that in the previous search [何] with the noisier 1998 data the cuts on $T_{\text {eff }}$ were made at between 25 and $100 \mathrm{mK}$.

From our previous experience we had learned that the detectors operate in a more stationary way when the noise temperature remains low for longer periods of time, because this indicates a smaller contribution of extra noise. To make a quantitative check on this we classified the data stretches in various categories, according to the length of the continuous periods having hourly averages $\overline{T_{\text {eff }}} \leq 10 \mathrm{mK}$, obtaining the figures shown in Table 2. From this table we clearly see that the longer is the time period of continuous operation with low noise the smaller is the number of events associated with a noise $T_{\text {eff }} \geq 7 \mathrm{mK}$.

Finally, in fig. 4 we show the distribution of the event energies selected according to $\overline{T_{e f f}} \leq 10 \mathrm{mK}$ and to $T_{\text {eff }} \leq 7 \mathrm{mK}$, for each event, belonging to periods with duration $\geq 1$ hour. We notice that, in spite of our selection criteria, we still have several events with large energy, which indicates the presence 

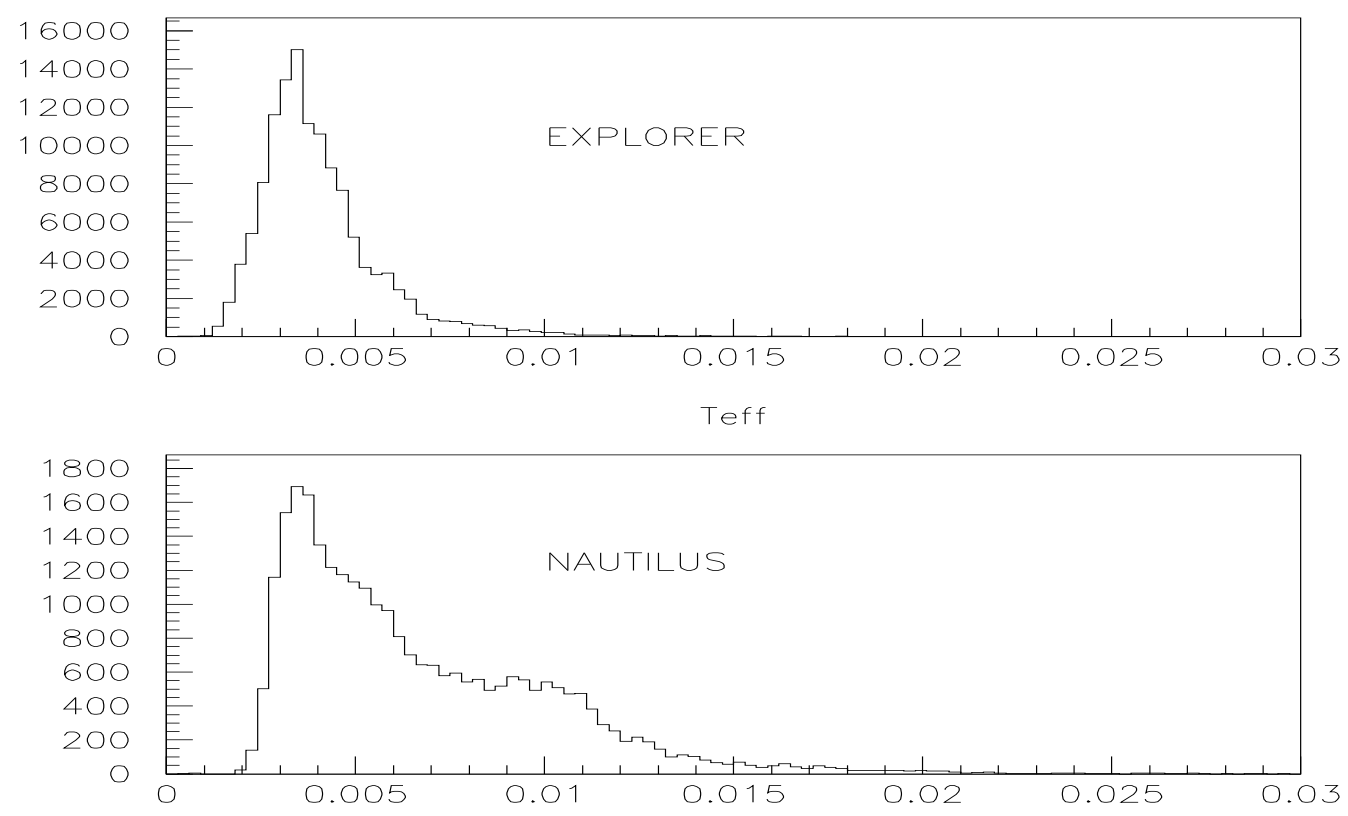

Fig. 3: The distributions of the ten minute averages of $T_{e f f}$, before each event, in kelvin units for the EXPLORER and NAUTILUS events (with hourly $\overline{T_{e f f}} \leq 10 \mathrm{mK}$ ). We accept only the events with the ten minute average $T_{\text {eff }}<7 \mathrm{mK}$. Note that the EXPLORER events are more numerous than the NAUTILUS events because of the different bandwidth.

Table 2: In the first column we indicate the minimum time length of continuous operation, in the second column the total common time of measurement. The last columns indicate the number of events, only those during the common time of operation, with hourly $\overline{T_{\text {eff }}} \leq 10 \mathrm{mK}$ and the percentage of the events having the ten minute average $T_{\text {eff }}>7 \mathrm{mK}$.

\begin{tabular}{|c|c|cc|cc|}
\hline time length & hours & EXPLORER & NAUTILUS & \\
& & events & $\%$ & events & $\%$ \\
\hline$\geq 1$ hour & 2156 & 54762 & 5.9 & 11252 & 37 \\
$\geq 3$ hour & 2082 & 52683 & 5.0 & 10887 & 34 \\
$\geq 6$ hour & 1927 & 50344 & 4.1 & 9939 & 31 \\
$\geq 12$ hour & 1490 & 40105 & 3.2 & 7268 & 27 \\
\hline
\end{tabular}



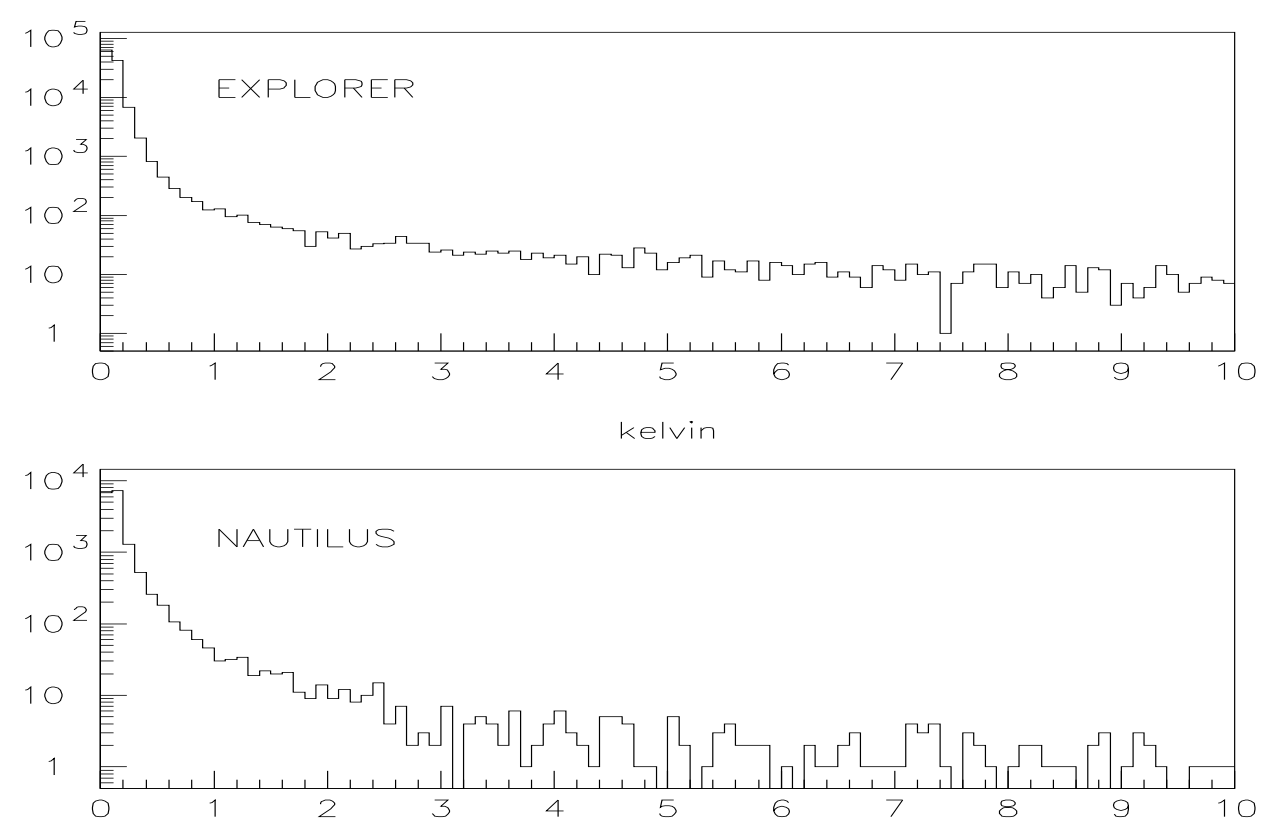

Fig. 4: The distributions of the EXPLORER and NAUTILUS event energies in kelvin units.

of extra noise, in addition to the thermal and electronic ones. The only way to eliminate this noise is by means of the coincidence technique.

\section{Searching for coincidences}

For the search for coincidences it is important to establish the time window. Using simulated signals and real noise, we characterized [10] the dispersion of the time of the event around the time when the signal is applied.

The standard deviation of the time dispersion for a given detector is

$$
\sigma_{d}=\operatorname{const} \frac{1}{\Delta f} \frac{1}{\sqrt{R_{e}}}
$$

for delta signals, where $\Delta f$ is the detector bandwidth and the const $=0.28$ is determined, for the EXPLORER and NAUTILUS detectors, by means of simulation [11]. For a coincidence analysis with two detectors we have

$$
\sigma_{w}=\sqrt{\sigma_{\text {expl }}^{2}+\sigma_{\text {naut }}^{2}}
$$

We decided to take as coincidence window $w= \pm 3 \sigma_{w}$, which is the most recent choice of the IGEC collaboration. Since each event has its own $\sigma_{w}$ the value of $w$ will be different for each coincidence. Note that the value of $w$ is almost entirely due to the NAUTILUS detector, since EXPLORER has a much larger bandwidth; it turns out to be of the order of $|w| \sim 0.5 \mathrm{~s}$, about one half of that used in previous searches for coincidences. With the use of $3 \sigma_{w}$ we also take into account the uncertainty of the detector bandwidth and of the simulation procedure.

Analysis in a coincidence search consists of comparing the detected number of coincidences at zero time delay $( \pm w)$ with the background, that is with coincidences occurring by chance. In order to measure the background due to the accidental coincidences, using a procedure adopted since the beginning of the gravitational wave experiments [12], one shift the time of occurrence of the events of one of the two detectors a number of times. We shifted 100 times in steps of $\Delta t=2 \mathrm{~s}$ (uncorrelated data), from $-100 \mathrm{~s}$ to $+100 \mathrm{~s}$. For each time shift we get a number of (shifted) coincidences. If the time 
shift is zero we get the number $n_{c}$ of observed coincidences. The accidental background is calculated from the average number of the $n_{\text {shift }}$ shifted coincidences obtained from the one hundred time shifts

$$
\bar{n}=\frac{\sum_{j=1}^{100} n_{\text {shift }}(j)}{100}
$$

This experimental procedure for evaluation of the background has the benefit of handling the problems arising when the distribution of the events is not stationary (see reference [13]), although this is not the case with the present 2001 data.

\section{Energy filter}

It is clear that if a coincidence between the two detectors is due to the arrival of a GW burst we expect the energies of the two coincident events to be correlated, and we can disregard all coincidences whose corresponding event energies are very different, according to the considerations illustrated in fig.1. Thus we can apply an energy filter with the aim of reducing the background.

The procedure for application of such an energy filter was set in our previous search for coincidences [7]. We considered signals of various energies $E_{s}$. For each coincidence found we calculated the $R_{s}$ for each of the above signal energy $E_{s}$ using the known values of the (local ) $T_{\text {eff }}$ of the two events. We then verified whether the two $R_{e}$, for the two events of that coincidence, fell within the interval $R_{s} \pm \Delta R_{s}$, such that the two limits $R_{s}-\Delta R_{s}$ and $R_{s}+\Delta R_{s}$ delimitate (see fig. 11) an area of $68 \%$ (about one standard deviation for well behaved noise) for a given value of $E_{s}$; that is, we verified the compatibility of the two events. We followed the same procedure for the shifted coincidences in order to estimate the background after application of the energy filter. In this way we reduced, for the 1998 data, the average number of accidental coincidences from $\bar{n}=223$ to $\bar{n}=51$.

This procedure is useful, in particular, if the two detectors have different sensitivity, as in the case of the 1998 data, and, consequently, the event thresholds and the event energies are also different. In the case of two detectors with comparable sensitivity, however, one could also consider to compare directly the energies of the coincident events. For the 2001 data, although in this year the two detectors had comparable sensitivities (see fig.2), we decided not to change the procedure used for the 1998 data. We considered $\mathrm{GW}$ signals of energy $E_{s}$, in a range covering the energies of our events, i.e. $E_{s}$ from $5 \mathrm{mK}$ to $1 \mathrm{~K}$ in steps of $5 \mathrm{mK}$, and accepted the coincidence (at zero delay or at a shifted time) if the two events fell within the above interval $R_{s} \pm \Delta R_{s}$.

\section{Sidereal time distribution}

In our previous search for coincidences [7] we took into consideration the non-isotropic response of the detector to a GW burst. We had reasoned that, since extragalactic GW signals should not be detected with the present detectors, possible sources should be located in our Galaxy, or in the Local Group. If any of these sources exist we should expect a more favorable condition of detection when the detectors are oriented with their axes perpendicular to the direction of the potential source, since the bar crosssection is proportional to $\sin ^{4}(\theta)$, where $\theta$ is the angle between the detector axis and the direction of the line joining it with the source. We did find a small coincidence excess when angle $\theta$ with respect to the Galactic Centre was larger than a certain value (see fig.3 of Ref.[7]). The inconvenience of this method is that, due to the poor statistics, the result has to be presented in an integral type graph, which makes it difficult to appreciate the real statistical significance of the data. Furthermore, hypotheses must be made on the location of the GW source.

In the present search for coincidences we extend the previous analysis as follows. We still make use of the directional property of the antenna cross-section. As the Earth rotates around its axis, during the day the detector happens to be variably oriented with respect to a given source at an unknown location. Thus we expect the signal to be modulated during the day; more precisely the modulation is expected 

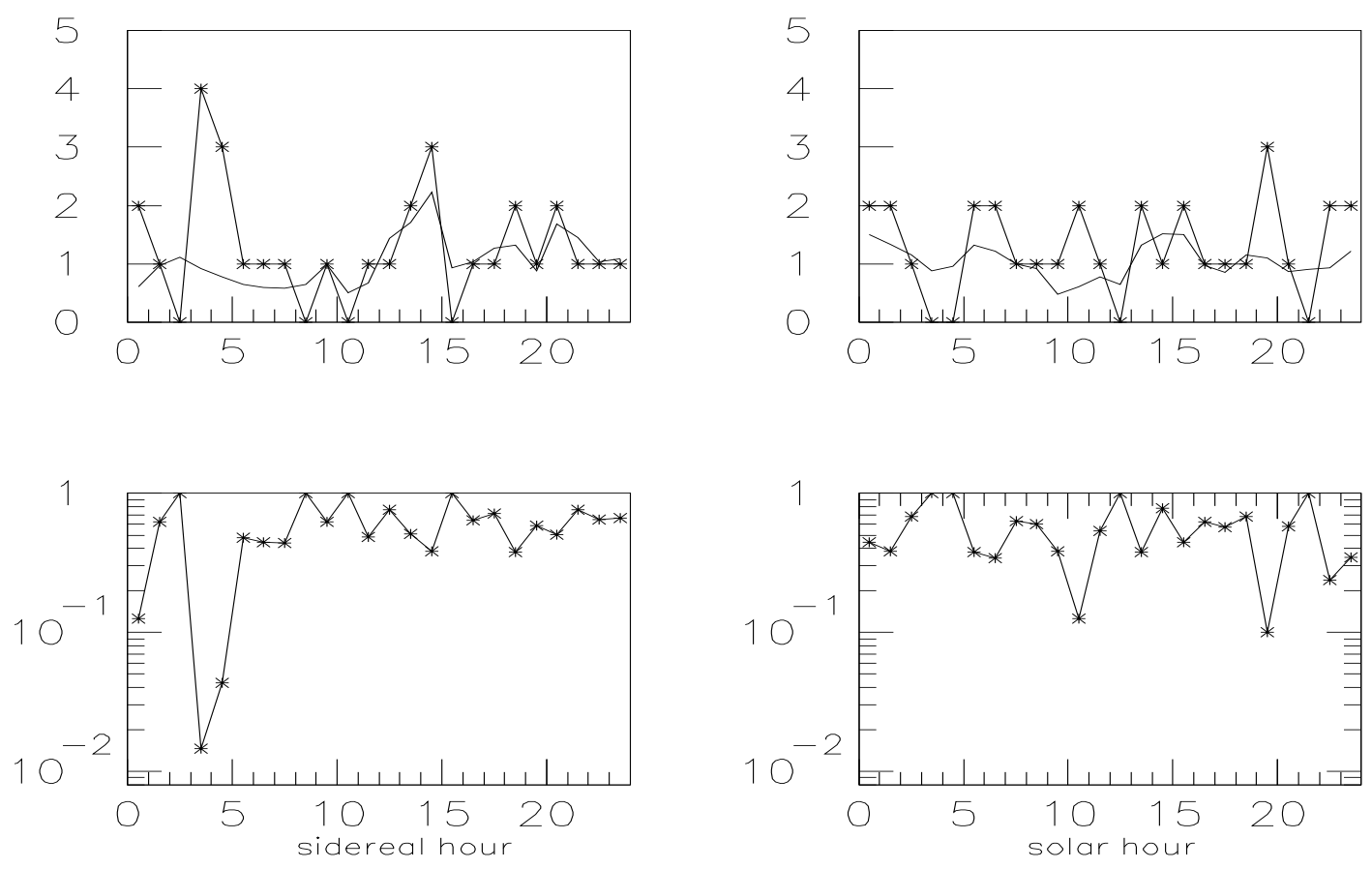

Fig. 5: Result with events in the long time periods ( $\geq 12$ hour ) of continuous operation. The upper graph on the left shows the number of coincidences $n_{c}$ indicated with the * and the average number $\bar{n}$ of accidentals versus the sidereal hour. The lower graph on the left shows the Poisson probability to obtain a number of coincidences greater than or equal to $n_{c}$. The two graphs on the right show the result using the solar time in hours. We remark that the data points refer to independent sets of events.

to have a period of one sidereal day (with one or more maxima) (see references [14, 15]), since the GW sources, if any, are certainly located far outside our Solar system.

The principal, key analysis is carried out with the events in the time periods of at least twelve hours of continuous data taking (see Table 2) to which the energy filter is applied.

Twenty-four categories of events are considered, one per each sidereal hour, the sidereal time referred to a position and orientation halfway between EXPLORER and NAUTILUS (this determines the zero local sidereal time which is not essential for the following considerations). Each category includes coincidences totally independent from those in the other categories. For each category in fig. 5 we report the number $n_{c}$ of observed coincidences, the average number $\bar{n}$ of accidental coincidences obtained by using the time shifting procedure and, given $\bar{n}$, the probability $p$ that a number $\geq n_{c}$ of coincidences could have occurred by chance. For comparison we also show a histogram produced with the same procedure using solar hours.

One notice a coincidence excess from sidereal hour 3 to sidereal hour 5, which appears to have some statistical significance, as the two largest excesses occur in two neighboring hours (the events in each hour are totally independent from those in a different hour). We have $n_{c}=7$ coincidences in this two-hour interval and $\bar{n}=1.7$. On the contrary, no significant coincidence excess appears at any solar hour.

The accidental coincidences always have a Poissonian distribution. To check this, we have considered for all the above events the accidental coincidences obtained with ten thousand trials, by time shifting from $-10000 \mathrm{~s}$ to $+10000 \mathrm{~s}$ in steps of two seconds. The distribution of the number of accidental coincidences is shown in fig. 6. The agreement between experimental and expected distributions is excellent.

We repeat the analysis for the events belonging to the larger set of continuous data taking lasting one hour or more (first line of Table22). We obtain the result shown in fig. П7. We notice that in the sidereal 


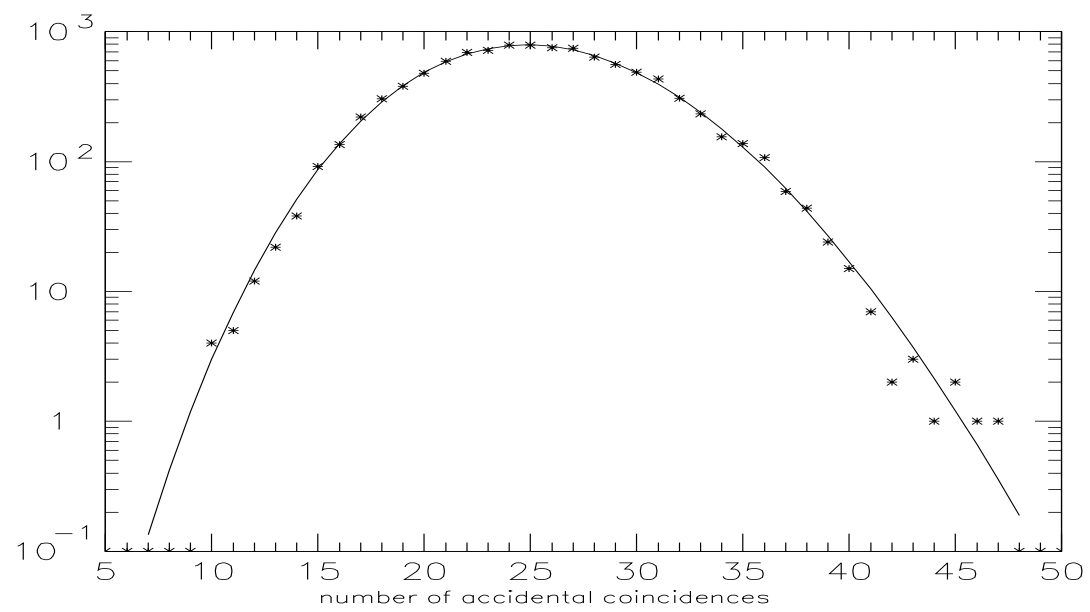

Fig. 6: The distribution of the number of accidental coincidences obtained with ten thousand trials, with average number $\bar{n}=25.32$. The stars indicate the experimental distribution, the continuous line the expected Poissonian distribution.
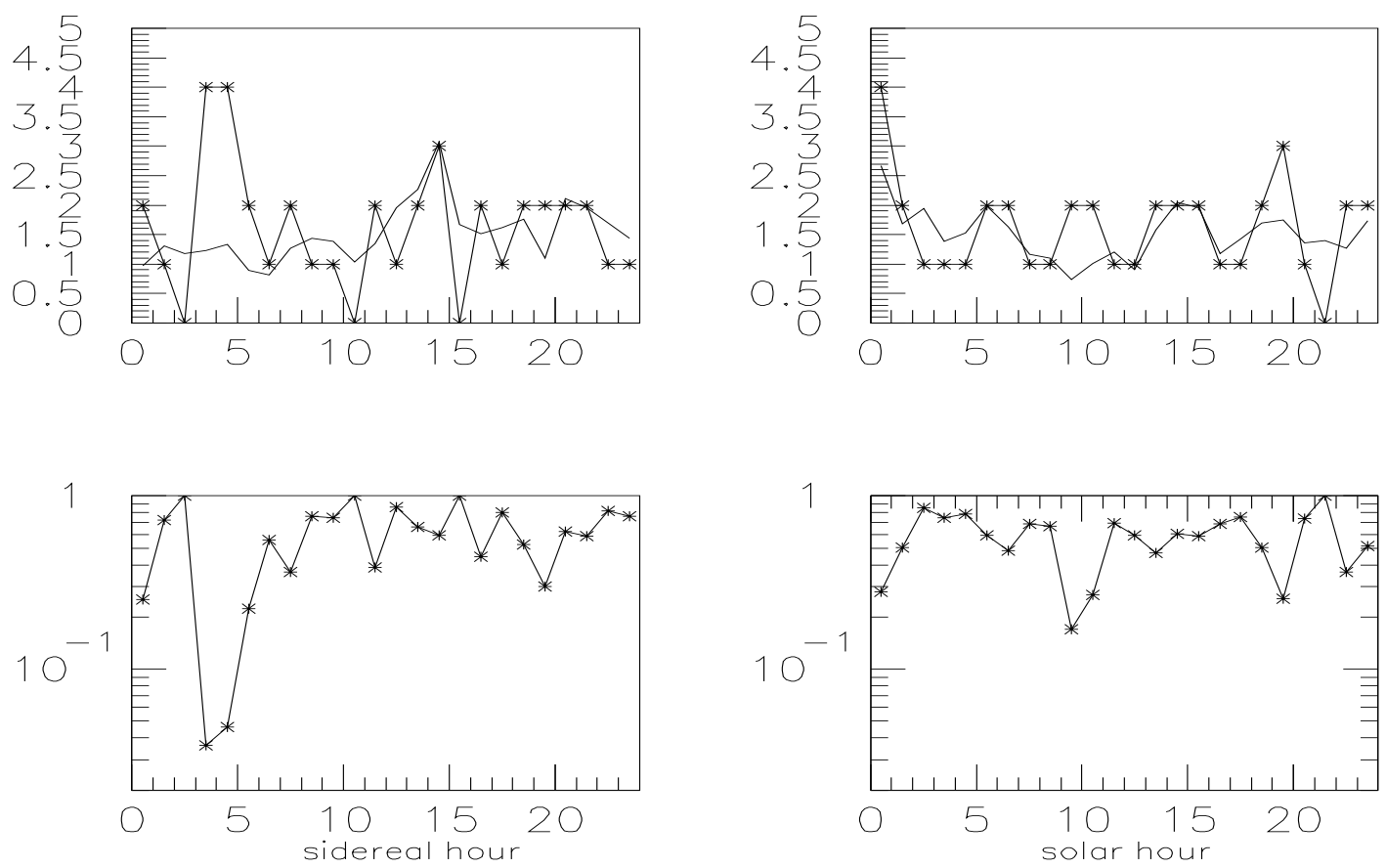

Fig. 7: Result with events in time periods $\geq 1$ hour of continuous operation. As in fig. 5 . 


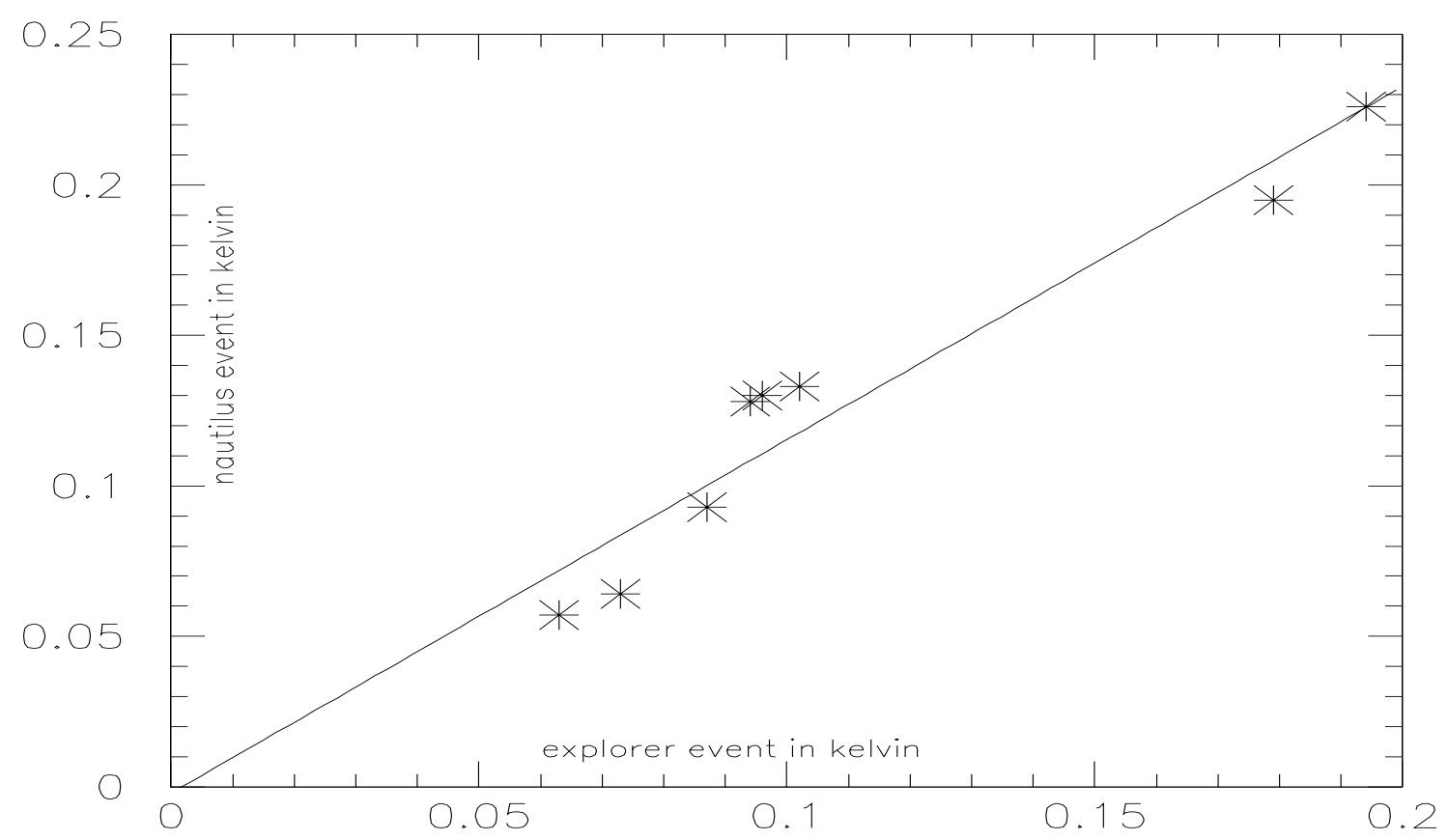

Fig. 8: Correlation between the event energies of NAUTILUS with those of EXPLORER for the eight coincidences occurred in the sidereal hour interval 3 to 5 , in time periods $\geq 1$ hour. The correlation coefficient is 0.96 . No energy filter was applied.

two-hour interval the number of coincident events has increased from seven to eight and the background has become $\bar{n}=2.6$.

We proceed now in performing a test, to check if this result is compatible with simultaneous physical excitation of the two detectors of possible non-terrestrial origin. We compare the energies of the coincident events: if the events in EXPLORER and NAUTILUS are due to the same cause we expect their energies to be correlated. This test must be done without applying the energy filter. Using the events in the time periods with duration $\geq 12$ hours and without applying the energy filter we still get seven coincidences. If we consider the time periods with duration $\geq 1$ hour we get eight coincidences. The event energies are very strongly correlated, as shown in fig.8. We also studied the energy correlation of the events of the accidental coincidences and found no correlation.

We also performed a coincidence data analysis when no energy filter at all was applied, obviously expecting a larger accidental background. The result is shown in fig. 9. We find that the coincidence excess in the time interval 3 to 5 sidereal hours still shows up, although less clearly, as expected.

A different way to present these data is shown in fig.10. This figure shows that at sidereal hours outside the interval 3 to 5 hours the event energies are not correlated. Also it shows the particular behaviour in the 3 to 5 hour interval, when the energies of all coincident events are correlated.

The eight events in the 3 to 5 hour period are listed in the Table 3 . We have verified that, using the cosmic ray detector of NAUTILUS, these events are not due to cosmic ray showers.

\section{Comparison with the $\mathbf{1 9 9 8}$ data}

The analysis presented here differs slightly (eg. for the use of the sidereal time) from that applied previously [7]. We therefore present the 1998 data also in terms of the sidereal time. We must consider that the 1998 data are noisier than the 2001 data. In particular the EXPLORER data have a noise $T_{\text {eff }}$ ten times larger than that of the 2001 data, whilst the NAUTILUS noise was of the same order.

For the coincidence search we change the window from $w= \pm 1 s$ (the IGEC choice at that time) used in the paper [7] to the present $w= \pm 3 \sigma_{w}$ used here. The result is given in fig.11. An effect similar 

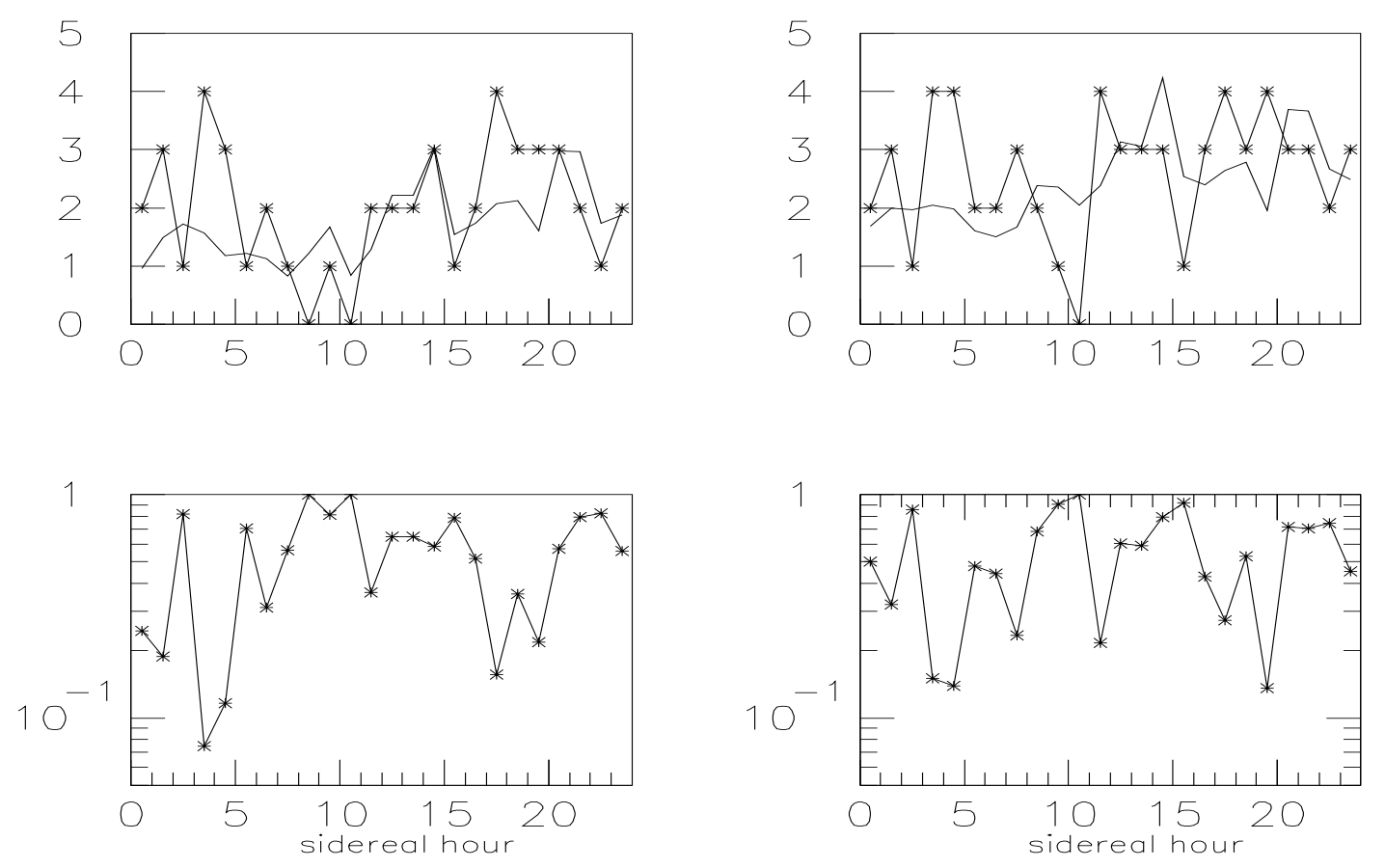

Fig. 9: As in fig. 5, with no application of the energy filter. The two graphs on the left refer to events in time periods with duration $\geq 12$ hours. The two graphs on the right refer to events in time periods with duration $\geq 1$ hour.

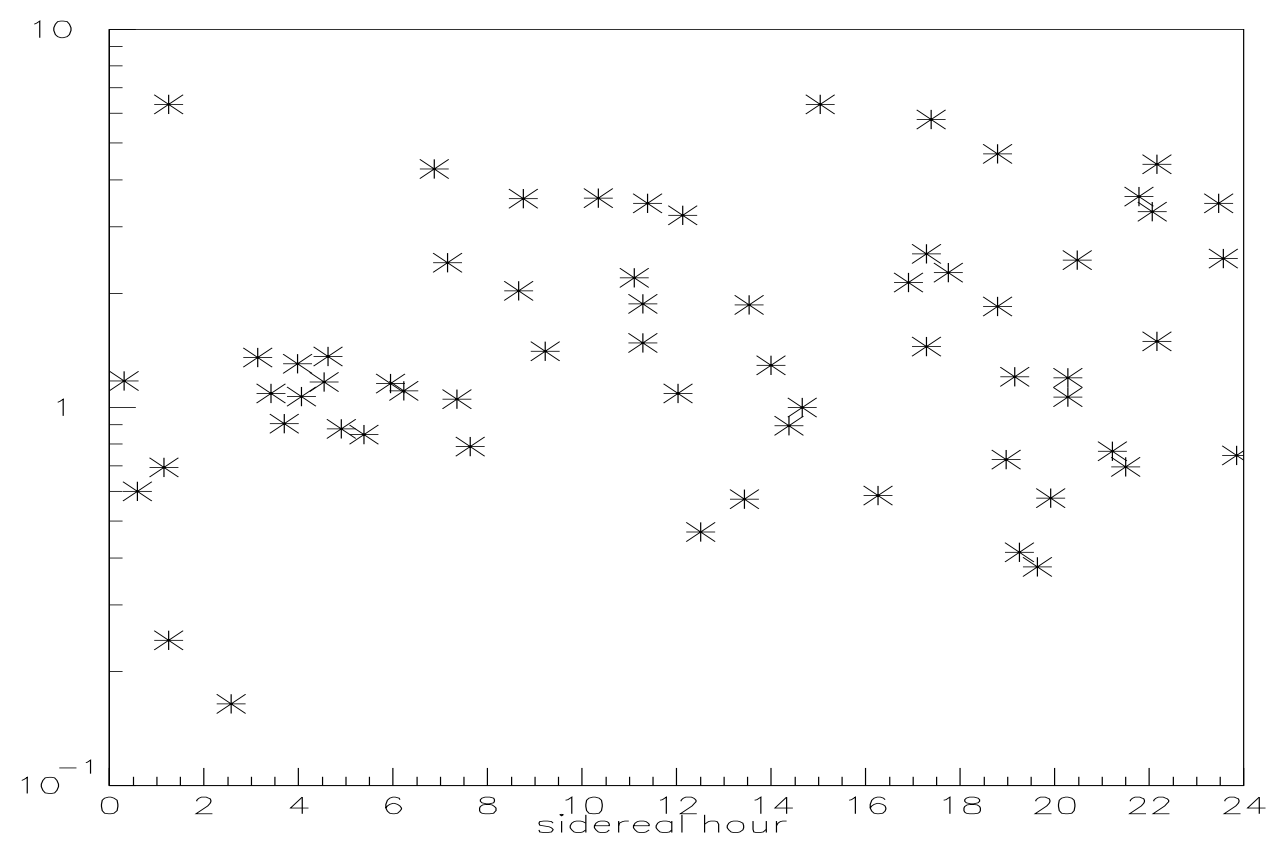

Fig. 10: Ratios of the energies (nautilus/explorer) for the events in coincidence belonging to periods with duration $\geq 1$ hour (no application of the energy filter) versus the sidereal hour. 
Table 3: List of the coincident events at sidereal hours between 3 and 5. No energy filter has been applied. $\delta t$ is the time difference between the two coincident events and $E$ is the event energy.

\begin{tabular}{|c|c|c|c|c|cc|cc|c|}
\hline day & hour & $\min$ & sec & $\begin{array}{c}\delta t \\
{[s]}\end{array}$ & $\begin{array}{c}\text { EXPLORER } \\
E\end{array}$ & $\begin{array}{c}{[m K]} \\
T_{\text {eff }}\end{array}$ & $\begin{array}{c}\text { NAUTILUS } \\
E\end{array}$ & {$[m K]$} & $\begin{array}{c}\text { sidereal } \\
\text { eff }\end{array}$ \\
hour \\
\hline 112 & 13 & 59 & 33.26 & 0.01 & 94 & 3.9 & 128 & 2.9 & 4.6 \\
130 & 11 & 39 & 28.07 & -0.08 & 179 & 3.6 & 195 & 5.9 & 3.4 \\
133 & 12 & 35 & 45.40 & -0.12 & 194 & 7.0 & 226 & 5.7 & 4.5 \\
166 & 10 & 48 & 6.04 & 0.39 & 73 & 3.2 & 64 & 3.0 & 4.9 \\
198 & 7 & 46 & 40.32 & 0.43 & 102 & 2.9 & 133 & 3.6 & 4.0 \\
278 & 2 & 12 & 29.65 & 0.37 & 63 & 2.9 & 57 & 2.6 & 3.7 \\
296 & 0 & 29 & 40.59 & -0.12 & 96 & 2.6 & 130 & 5.6 & 3.1 \\
296 & 1 & 24 & 10.46 & 0.00 & 87 & 2.8 & 93 & 4.1 & 4.1 \\
\hline
\end{tabular}
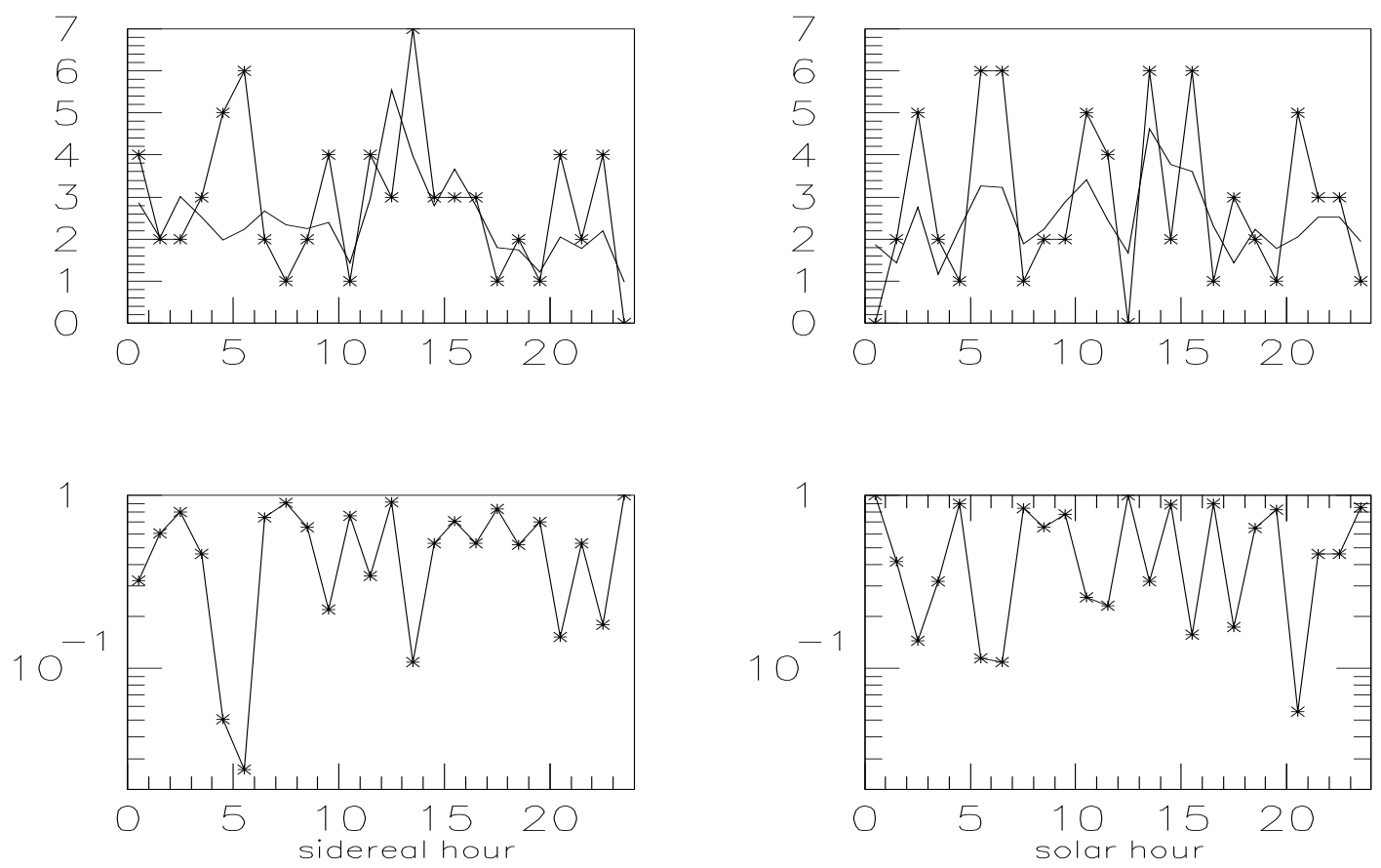

Fig. 11: 1998 data with energy filter. The upper graph on the left shows the number of coincidences $n_{c}$ indicated with the * and the average number $\bar{n}$ of accidentals versus the sidereal hour. The lower graph on the left shows the Poisson probability of obtaining a number of coincidences greater or equal to $n_{c}$. The two graphs on the right show the result using the solar time in hours. 

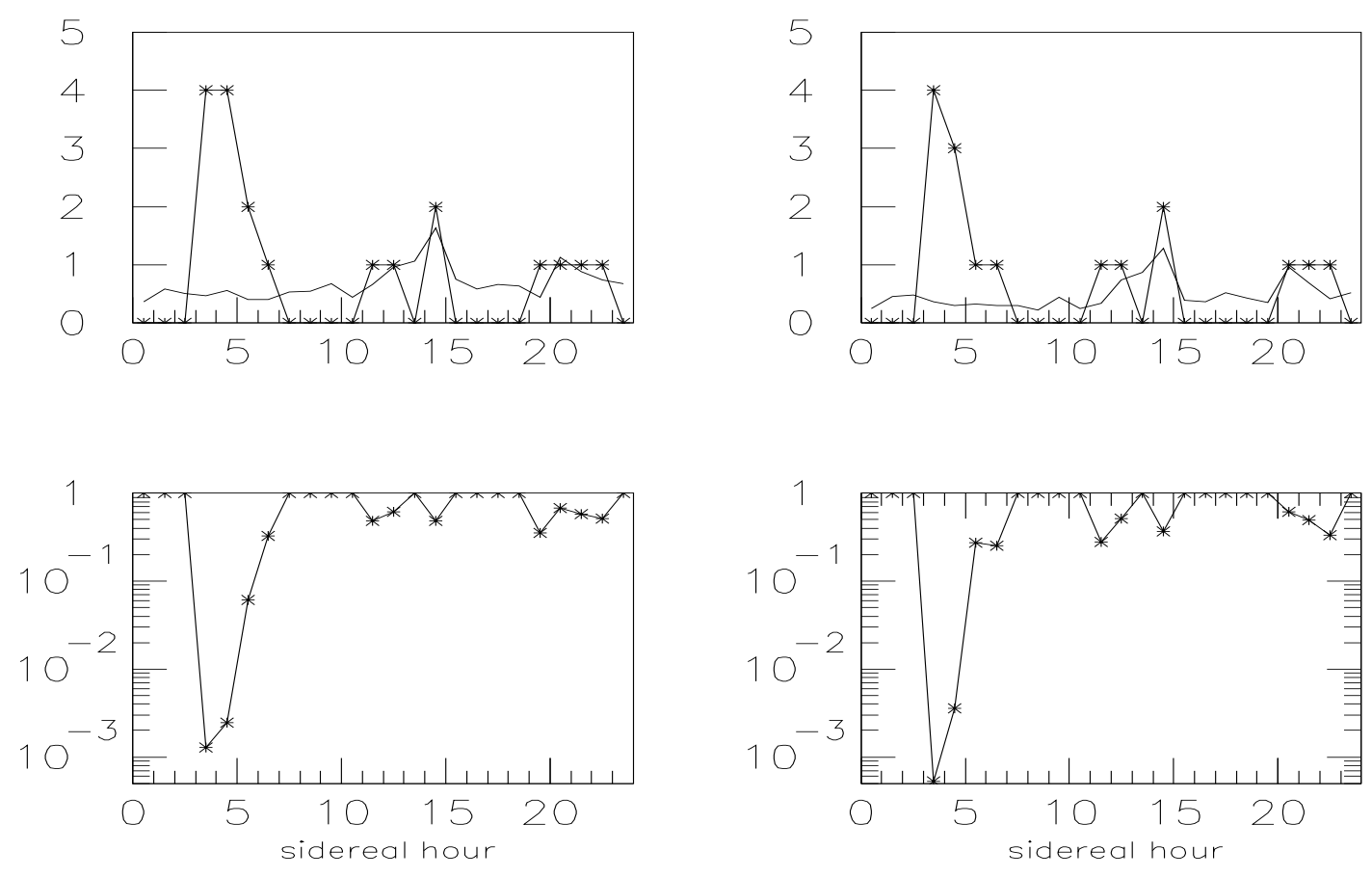

Fig. 12: 2001 data with "a posteriori" choices (see text). As in fig 7 for the two graphs on the left (periods $\geq 1$ hour) and as in fig. 5 for the two graphs on the right (periods $\geq 12$ hour). Again we remark that the data points in the figure at different sidereal hours are independent one from the other.

to the one found for the 2001 data is noticed, although weaker than that obtained with the less noisy 2001 data.

\section{Robustness of the statistical analysis}

In any data analysis care must be taken to avoid choosing procedures which favour particular results. Thus we considered the possibility that our result be biased, although involuntarily, by any such choices. In the present analysis all choices were made "a priori" and already published in the scientific literature; in particular the IGEC choice for the coincidence window of $\pm 3 \sigma_{w}$, the energy filter (see ref. [1] and [7]) and the threshold for definition of an event (see ref.[6]).

Nevertheless, we tested whether our present choices were indeed, to accident, most apt to produce the coincidence excess. This proved not to be the case.

To find the most favorable parameters, that is the threshold and the energy filter parameter, we considered only the coincidences in the sidereal hour interval 3 to 5 and minimized the probability of a coincidence excess by chance. We found that the most favorable threshold for the definition of event is at $R_{t}=20.5$, instead of 19.5 . The most favorable parameter for the energy filter is $50 \%$, instead of $68 \%$.

We thought it interesting to report the result for these most favorable choices, $R_{t}=20.5$ for the threshold and $50 \%$ for the energy filter. The result is shown in fig.12. We notice an indication that the coincidence excess might extend to the sidereal hour interval 3 to 6 , including two more coincidences in the period 5 to 6 sidereal hours (see also fig.10). We want to remark that this hour was not included in the optimization process.

As far as the coincidence window is concerned, we found (a posteriori) that the best choice for having a coincidence excess in the 3 to 5 sidereal hour interval is $\pm 3.5 \sigma_{w}$, and any coincidence window from $\pm 2.5 \sigma_{w}$ to $\pm 4 \sigma_{w}$ gives comparable results. 


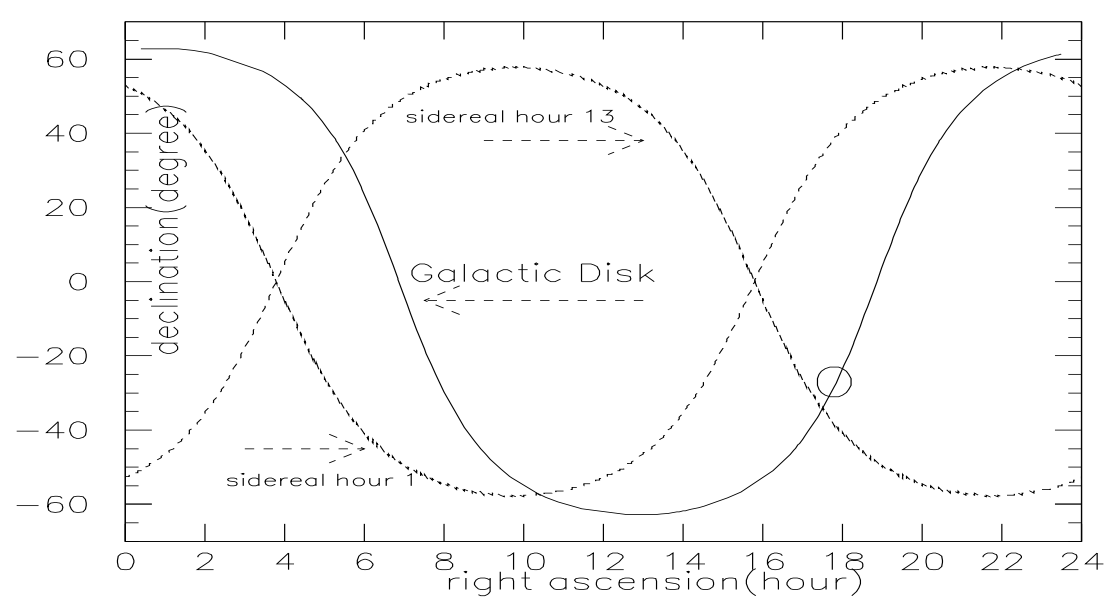

Fig. 13: The position of the Galactic Disk and the loci of point sources perpendicular to the detector axis at 1 and 13 sidereal hour in the right ascension-declination plane. At sidereal hour 4.3 the locus tends to coincide with the line of the Galactic Disk. The large circle indicates the location of the Galactic Centre.

\section{Discussion and conclusions}

The IGEC search for coincidences [6] was performed without applying the event energy algorithms based on the event amplitude and on the directional properties of the detectors; it gave a no coincidence excess. With new data taken in the year 2001 and with improved sensitivity we repeated the coincidence search with the detectors EXPLORER and NAUTILUS (no other detector was in operation during the year 2001), applying data analysis algorithms based on known physical characteristics of the detectors, namely energy of the events and directionality of the detectors. We obtained a coincidence excess at sidereal hours between 3 and 5 .

At a given sidereal time the intersection of the celestial sphere with the plane perpendicular to the detector axis is a circle. We show in fig. 13 two of these circles (i.e., at 1 and at 13 sidereal hours) in the right ascension-declination plane. In the representation of fig. 13 the line which indicates the point sources perpendicular to the detector axis (we call this the line of maximum sensitivity) moves to the right with sidereal time. This line intersects the location of the Galactic Centre twice a day (at 4.3 and at 13.6 sidereal hours). Only once per day (at 4.3 sidereal hour) this line overlaps with the entire Galactic Disk. The overlapping takes place because of the particular orientation of the detectors on the Earth' surface (see Table 1), and would not occur for a different azimuth angle of detector orientation.

If all GW sources were concentrated in the Galactic Centre we would have found a coincidence excess twice a day. The coincidence excess occurs only once per day, just when the line of maximum sensitivity of the detectors overlaps with the Galactic Disk, as if GW sources were distributed in the Galactic Disk and not just located in its Center.

As for the energy balance, in the year 2001 we find in the interval from 3 to 5 sidereal hour a coincidence excess of, very roughly, $n_{c}-\bar{n} \sim 6$ coincidences occurring in five days (two sidereal hours out of twentyfour, in a total time period of 1490 hours $\sim 60$ days). In terms of energy conversion into $\mathrm{GW}$ we have, very roughly, about one coincidence per day with a signal energy of about $100 \mathrm{mK}$. This corresponds, using the classical cross-section, to a conventional burst with amplitude $h \sim 210^{-18}$ and 
to the isotropic conversion into GW energy of 0.004 solar masses, with sources located at distance of 8 $\mathrm{kpc}$. The observed rate is much larger than the models today available predict, for galactic sources. We note, however, that our rate of events is within the upper limit determined by IGEC [6] for short GW bursts and by the 40m-LIGO prototype interferometer [16] for coalescing binary sources in the Galaxy.

We think it is unlikely that the observed coincidence excess be due to noise fluctuations, but we prefer to take a conservative position and wait for a stronger confirmation of our result, before reaching any definite conclusion and claim that gravitational waves have been observed. Furthermore, although we have excluded that the events are due to cosmic ray showers (see section 6), we cannot completely rule out that they be due to some other exotic, still unknown, phenomenon. A possible way to distinguish GW from other causes is to measure other vibrational modes of the detectors and verify that, as predicted by General Relativity, only quadrupole modes are excited. This requires multimode detection (with bars or spheres) and to improve the signal-to-noise ratio of the apparatuses.

We expect to collect new data with EXPLORER and NAUTILUS with improved sensitivity. We plan to repeat the same analysis with these new data and also with any other new data provided by other GW groups, those which operate the resonant detectors and those which operate or are beginning to operate the interferometric detectors GEO, LIGO, TAMA and VIRGO.

\section{Acknowledgements}

We are indebted to Professor Ugo Amaldi for very useful discussions and suggestions. We thank the CERN cryogenic facility and F. Campolungo, R. Lenci, G. Martinelli, A.Martini, E. Serrani, R. Simonetti and F. Tabacchioni for precious technical assistance.

\section{References}

[1] P. Astone et al., Phys. Rev. D. 47, 362 (1993).

[2] E. Mauceli et al., Phys.Rev. D, 54, 1264 (1996)

[3] D.G. Blair et al. Phys. Rev. Lett.74, 1908 (1995).

[4] P. Astone et al, Astroparticle Physics, 7, 231(1997)

[5] M.Cerdonio et al., Class.Quant.Grav.14(1997) 1491-1494

[6] Z.A. Allen et al. Phys.Rev.Lett.85(2000) 5046-5050

[7] P.Astone et al. Class. Quantum Grav. 18(2001) 243-251

[8] P.Astone, C.Buttiglione,S.Frasca, G.V.Pallottino, G.Pizzella Il Nuovo Cimento 20, 9 (1997)

[9] A.Papoulis "Probability, Random Variables and Stochastic Processes", McGraw-Hill Book Company (1965), pag 126.

[10] P.Astone et al. Phys.Rev. D 62(2000) 042001

[11] P.Astone et al.,"Adaptive matched filters for the EXPLORER and NAUTILUS gravitational wave detectors" in preparation (2002)

[12] J. Weber, Phys. Rev. Lett. 22, 1320 (1969).

[13] P.Astone, S.Frasca, G.Pizzella, Int.J.Mod.Phys.D9(2000) 341-346

[14] J.Weber, Phys. Rev. Letters 25(1970) 180

[15] Yu.V. Baryshev and G. Paturel, e-Print Archive: astro-ph/0104115 (April 2001) 
[16] B.Allen et al., Phys. Rev. Lett. 83, 1498 (1999) 